\title{
Initial presenting symptoms and severity of SARS-CoV-2 Wild type, the Delta variant and the Omicron variant infected cases in early fourth wave of epidemics in Myanmar
}

Khin Phyu Pyar ${ }^{1 *} \mid$ Khine Khine $\mathrm{Su}^{2} \mid$ Kyaw Wunna ${ }^{3} \mid$ Zar Ni Htet Aung ${ }^{4} \mid$ Nyan Lin Maung ${ }^{4} \mid$ Aung Phyoe Kyaw ${ }^{4} \mid$ Soe Win Hlaing ${ }^{4} \mid$ Thi Han Tun ${ }^{6} \mid$ San Myint Htun ${ }^{7}$ Nay Myo Aung ${ }^{7} \mid$ Aye Min Than ${ }^{7} \mid$ Min Kyi Mon ${ }^{6} \mid$ Win Min ${ }^{7} \mid$ Thein Tun Myint ${ }^{4}$ Kyaw Zay Ya ${ }^{4} \mid$ Thurein Win ${ }^{4} \mid$ Min Aung Shan ${ }^{5} \mid$ Si Phyo Thu ${ }^{5} \mid$ Yan Lin Aung ${ }^{5}$ Zay Phyo Aung ${ }^{4}$ |Myo Thant Kyaw ${ }^{4} \mid$ Kyaw Thet Maung ${ }^{4} \mid$ Han Lin Aung ${ }^{4}$

\footnotetext{
${ }^{1}$ Professor and Head/Senior

Consultant Physician,

Department of Medicine/

Department of Nephrology,

Defence Services Medical

Academy, No. (1) Defence Services General Hospital (1000-Bedded)

${ }^{2}$ Professor and Head, Department of Microbiology, Defence Services Medical Academy

${ }^{3}$ Consultant Microbiologist, Department of Microbiology, No. (1) Defence Services General Hospital (1000-Bedded)

${ }^{4}$ Consultant Physician, No. (1) Defence Services General Hospital (1000-Bedded)

${ }^{5}$ Public Health Specialist, No. (1)

Defence Services General Hospital (1000-Bedded)

${ }^{6}$ Consultant Microbiologist, Department of Microbiology, Defence Services Medical Academy

${ }^{7}$ Consultant Physician, Defence Services Liver Hospital (300-Bedded)
}

\section{Abstract}

Copyright : (C) 2022 The Authors. Published by Medical Editor and Educational Research Publishers Ltd. This is an open access article under the CC BY-NC-ND license (https://creativecommons.org/licenses/by-nc-nd/4.0/). 


\section{1 | INTRODUCTION}

$\mathrm{T}$ he "severe acute respiratory syndrome coronavirus type 2" (SARSCoV-2) is also known as "coronavirus disease 19" (COVID-19). It originated in Wuhan, Hubei province, People's Republic of China, in December 2019; and, it spread worldwide causing a global pandemic. Genetic variants of SARS-CoV-2 have been emerging and circulating around the world throughout the COVID19 pandemic till 2022: original (wild-type), Alpha, Beta, Delta, and Gamma. In third wave, the notorious variant was the Delta variant; it was well-known for quick transmissibility, causing high morbidity and mortality. In late November, the Omicron variant was first detected in South Africa. Therefore, evidences are required about the transmissibility and virulence among several variants and old Wild type.

The transmissibility of the Omicron variant compared to other variants is not clearly known (World Health Organization, 2021). The study from Norway, Brandal et al., (2021) revealed that the SARSCoV-2 Omicron variant was highly transmissible even among fully vaccinated young and middle-aged adults. The question of "whether the Omicron variant was imported from other/neighboring countries or it mutated from the remining Wild type in our own country, Myanmar" was very difficult. Moreover, one study proved that the Omicron variant originated from mouse (Wei et al., 2021). The transmission from western part of world/neighboring countries was also possible. The omicron variant was at least twice as contagious as delta and at least four times as contagious as the original version of the coronavirus (UK Health Security Agency, 2021). A new study on recent Ontario COVID-19 cases suggests the Omicron variant is less likely to cause hospitalization or death than the Delta variant, but could still significantly impact health-care systems due to its high transmissibility (Public Health Ontario, 2021).

The reported symptoms with the Delta variant were headache, aches and pain, sore throat, runny nose, fever and cough (Pyar et al., 2021). Running nose, sneezing (stuffy nose), sore throat, fatigue and headache were top 5 symptoms found with the Omicron variant infected persons, reported by Zoe Covid
Study Group (2022). They also found that the symptoms of the Omicron variant infected persons were not statistically different from those infected with the Delta variant where only $50 \%$ of people experiencing the classic three symptoms of fever, cough, or loss of sense of smell or taste. According to UK Health Security Agency (2021), sore throat was seen in half of the cases with the Omicron variant; however, it was noted in one-third of the cases with the delta variant. They also compared the symptoms; a loss of smell and taste was less common among omicron cases compared to delta variant cases.

Regarding the clinical severity of the variant, patients infected with the Delta variant had moderate to severe status with variable mortality depending mainly on clinical severity and co-morbid status. Of clinical severity of the Omicron variant, the reports were contradictory. The preliminary data from South Africa suggested that not only the number of the Omicron infected cases but also the number of cases to keep in hospital were high; the cases were reported as severe (World Health Organization, 2021). On the other hand, in Scotland, early national data revealed that the Omicron variant was associated with a twothirds reduction in the risk of COVID-19 hospitalisation when compared to Delta variant (Sheikh et al., 2021). Hospitalisation risk from Omicron infection was nearly one-third of Delta (UK Health Security Agency, 2021). The researchers from California also pointed out that the Omicron infected cases were less severe (Lewnard et al., 2022); and, it was proved by one preprint study (Wang et al., 2022). Omicron infections were associated with a $91 \%$ reduction in risk of death compared to the Delta variant (Lewnard et al., 2022).

According to the vaccination record, nearly half of the world population had completed vaccination

Supplementary information The online version of this article (https://doi.org/10.52845/JMRHS/20225-1-8) contains supplementary material, which is available to authorized users.

Corresponding Author: Khin Phyu Pyar

Professor and Head/Senior Consultant Physician, Department of Medicine/ Department of Nephrology, Defence Services Medical Academy, No. (1) Defence Services General Hospital (1000-Bedded) 
(World Health Organization, 2022). In Myanmar, in early January 2022, nearly $30 \%$ of population were fully vaccinated, according to report from Ministry of Health. The morbidity and mortality of SARSCoV-2 Wild type, Delta variant and Omicron variant infected cases may be better with increasing COVID19 coverage. The study from U.K pointed out that "the third/booster dose of vaccination offers substantial additional protection against the risk of symptomatic COVID-19 for being infected with the Omicron variant when compared to $\geq 25$ weeks post second vaccine dose" (Sheikh et al., 2021). In the outbreak report from Norway, the SARS-CoV-2 Omicron variant was highly transmissible among fully vaccinated young and middle-aged adults (Brandal et al., 2021). Hansen et al., (2021) found that the Omicron variant invaded COVID-19 vaccine more than the Delta variant.

Based on WHO severity score, the clinical severity of COVID-19 infection was classified into four types: mild, moderate, severe and critical. In mild category, patients have symptoms only, CXR is normal and, $\mathrm{SaO}_{2}$ on air is normal. In moderate category, CXR shows pneumonias and $\mathrm{SaO}_{2}$ on air is $\geq 90 \%$. In severe category, respiratory rate is $>30 / \mathrm{min}$ and, $\mathrm{SaO}_{2}$ on air is $<92 \%$. In critical disease category, the patient has ARDS; he may have sepsis with multi-organ dysfunction or septic shock or acute thrombosis (pulmonary embolism, acute coronary syndrome, acute stroke).

The clinical presentations and severity of SARSCoV-2 Wild type, the Delta variant and the Omicron variant infected cases in early fourth wave of epidemics in Myanmar was interesting; no report so far. Nasopharyngeal swabs were taken from both clinically suspicious cases, contacts of COVID-19 PCR positive cases, and healthy travelers coming to Myanmar at Mingaladon airport, No. (1) Defence Services General Hospital (1000-Bedded) and Mingaladon COVID-19 treatment Hospital (300Bedded) from October 2021 to early January 2022; then, they were proceeded with both Abbot COVID19 Antigen Rapid Test Device and RT-PCR. Then, they were differentiated with special tests for variant. Nasopharyngeal swabs were collected using plastic swab with nylon flocked swabs, it was placed in a $3 \mathrm{ml}$ viral transport media (Himedia, India). The virus RNA was extracted using the magnetic beads method, according to the instruction of the nucleic acid extraction kit (Bioer MagaBioplus Virus DNA/RNA purification Kit II, China). SARS CoV2 RNA detection was done by bioPerfectus Nucleic Acid Detection Kit (bioPerfectus, Jiangsu bioPerfectus Biotech Co.,Ltd, China). All SARS CoV-2 positive samples were tested with abTES ${ }^{\mathrm{TM}}$ COVID-19 Variant qPCR I kit (AIT biotech Pte-Ltd, Singapore), using Applied Biosystem 7500 Fast Real Time PCR System according to the manufacturer's instruction. abTES ${ }^{\mathrm{TM}}$ COVID-19 variant qPCR I kit differentiates wild and variant SARS CoV-2 infection among all positive samples. After that, SARS CoV-2 Alpha, Beta, Gamma, Delta \& Omicron variant infection among all SARS CoV-2 variant samples were tested by GenXPro SARS CoV-2 ABGD variant Detection Kit (GenXPro, Germany) and SARS CoV-2 variant Omicron (B.1.1.529) Real Time PCR Kit (bioPerfectus, Jiangsu bioPerfectus Biotech Co.,Ltd, China). SARS CoV-2 variant Omicron (B.1.1.529) Real Time PCR Kit detect Orflab gene and mutations E484A, N679K, L981F, 69-70del and H655Y of $\mathrm{S}$ gene. A sample was considered as Omicron (B.1.1.529) if any two of the three specific targets (E484A, N679K and L981F) with cycle threshold (Ct) less than 40 and $\Delta \mathrm{Ct}$ values were detected in manufacturer's reference range.

Clinical severity/symptoms, travel history, vaccination history and co-morbid status was taken either face to face (if they came to No (1) DSGH or Mingaladon COVID-19 treatment Hospital (300 Bedded)) or viper/telecommunication (if they did not come to No (1) DSGH or Mingaladon COVID-19 treatment Hospital (300 Bedded). Then, clinical, chest radiograph (mild, moderate and severe cases) and molecular parameters were analyzed. Those requiring hospital admission at Mingaladon COVID-19 treatment Hospital (300 Bedded) were treated according to hospital/National guideline: antiviral drugs, antibiotics, oxygen therapy, steroid therapy, heparin therapy, fluids and electrolyte and supportive care. The blood tests were done according to guideline and monitored till discharge from Mingaladon COVID19 treatment Hospital (300 Bedded) or death. 


\section{MEERP LTD}

During this period, among 50,842 nasopharyngeal swab samples were tested and $770(1.5 \%)$ samples were SARS CoV-2 test positive. The total number of positive samples on October, November, December and January were 321, 272, 149 and 28 respectively. In these 770 positive samples, $\mathrm{Ct}$ value less than 30 were selected $(\mathrm{n}=150)$. Table (1) Wild type was seen in 2 cases $(2 / 150=1.3 \%)$ and Delta variant 138 cases $(138 / 150=92.0 \%)$. The Omicron variant was detected only in January 2022; the number was 10 $(10 / 150=6.7 \%)$ over five months.

Regarding clinical presentations, the Omicron variant caused more of upper respiratory symptoms as its main habitus was upper airways. Thus, the mortality rate was relatively low than the Delta variant. The Delta variant produced severe/critical manifestation; and, the mortality was the highest among all the variants. The wild type had the lowest infectivity and mortality among SARS CoV-2 virus.

There were two cases infected with the Wild type in this study; the first case was fully vaccinated and the second case had incomplete vaccination. Their symptoms were fever, loss of smell and cough. The finding was comparable with the earlier study done in second wave of epidemics in Myanmar at the end of 2020, probably the wild type; it revealed that $81.5 \%$ were symptomatic patients the most common presenting symptoms were fever $54.1 \%$, loss of smell $50.3 \%$, and cough $30.9 \%$ (Htun et al., 2021). As a severity, $20.7 \%$ of patients had signs of severe pneumonia; however, in this study, both were mild form and not fatal.

In this study, the Delta variant infected the majority of cases (92\%); it produced mild $36.2 \%$ (50 cases), moderate $52.2 \%$ (72 cases), severe $10.9 \%$ (15) and critical $0.7 \%$ (1) infection. The common presentations in this study were fever $(40 \%)$, dyspnea $(30 \%)$, cough $(20 \%)$, loss of smell $(20 \%)$, loss of taste $(13 \%)$, myalgia (13\%), sore throat $(13 \%)$, runny nose $(13 \%)$, and loose motion (13\%). The findings were comparable with the study by Pyar et al., (2021) in Myanmar. They did the study in the third wave of epidemics in Myanmar mid 2021 where the strain was probably the Delta variant; the top symptoms seen among physicians with break through infections were a headache, sore throat, runny nose, fever and cough (Pyar et al., 2021).

The number of cases required hospital admission was $88(63.8 \%)$, all were infected with the Delta variant; moderate, severe and critical cases. The mortality rate was $5.8 \%$ ( 7 severe and one critical case): COVID-19 related death in 6 cases and COVID-19 unrelated death in 2 cases.

The cause of death was related to COVID-19 in 6 cases. The one and only one critical case was deeply comatose (Glasgow Coma Scale 3/15) at the time of admission; associated co-morbidities were old age (63 year), hypertension with moderate cardiomegaly, diabetes mellitus, acute kidney injury (creatinine 1.8 $\mathrm{mg} \%$ ), very high $\mathrm{D}$ dimer (> 10,000 $\mathrm{ng} / \mathrm{ml}), \mathrm{SaO}_{2}$ $85 \%$ on air and right upper lobe collapse in chest radiograph. Two out of six fatal cases had COVID19 related gastrointestinal complications. One case had gastrointestinal hemorrhage, thrombocytopenia (platelet count $20 \times 10^{9} / \mathrm{L}$ ) and hypothyroidism; and another case had duodenal perforation, age 65 , died on $4^{\text {th }}$ post-operative day. The last fatal case was female, 47 years having many co-morbid conditions such as brain tumor, Atrial Septal Defect and diabetes mellitus; she died of COVID-19 pneumonia.

The cause of death was unrelated to COVID-19 in 2 cases: acute myeloblastic leukemia, age 33 year; and, cerebrovascular hemorrhage (Basal ganglia hemorrhage, 70-year male, died on post-operative day 4 following Burr Hole operation).

Although we have been trying to trace the Omicron variant since October 2021, it was not detected until end of December 2021. We discovered the Omicron only in early January 2022 in imported cases only i.e., travelers. These imported cases were coming from neighboring countries; 9 from India and 1 from Russia. Table (1) They were asymptomatic cases; and, they had co-morbidities like hypertension and diabetes mellitus which were well controlled. According to the UK study, a sore throat was commonly reported by the omicron virus cases. A loss of smell and taste was less common among omicron cases compared to delta variant cases. In this study, cases infected with the Omicron variant were asymptomatic; and, they did not need hospital stay or oxygen. Eighty percent of them were fully vaccinated. Only one case completed booster dose. 
With the development of vaccine, the break through infection following complete vaccination were reported from various studies. In this small study, half of cases with the Delta variant infection did not received vaccination. Most of the cases with the Omicron variant had completed vaccination. Like the Delta variant, the breakthrough infection could occur with the Omicron variant except two cases; parents and son aged 4 years with congenital heart disease, returned from India. They did not get the vaccine; however, they were asymptomatic. Like the Delta variant, the breakthrough infection could occur with the Omicron variant.

There were still imported cases of the Delta variant from neighboring countries. All the cases infected with the Omicron variant were imported too.

It can be concluded that the wild type was still infectious; however, it caused mild infection if there was no co-morbidity. Over sixty percent of cases infected with the Delta variant were moderate to severe/critical status and they required hospitalization. Nearly $6 \%$ of cases with the Delta variant were fatal. All the Omicron infected cases were asymptomatic; it was imported from neighboring countries. The breakthrough infection cases following completed vaccination were seen with both the wild type, and the Delta variant and the Omicron variant. Thus, we need to encourage vaccination program. Early and effective quarantine of asymptomatic cases with the Omicron variant may prevent further spread; key player in control of epidemics in Myanmar. Imported cases carry both the variant; Delta and Omicron. Although these data represent a sample from a region and it highlighted the awareness among all health care personnel and publics particularly screening at entry points to Myanmar.

Table (1) Clinical severity status of cases infected with different variant

\begin{tabular}{|l|r|r|r|r|r|r|r|l|l|}
\hline $\begin{array}{l}\text { Type of } \\
\text { SARS } \\
\text { CoV-2 } \\
\text { virus }\end{array}$ & $\begin{array}{r}\text { Number } \\
\text { of cases }\end{array}$ & $\begin{array}{l}\text { Asymp- } \\
\text { tomatic }\end{array}$ & Mild & Moderate & Severe & Critical & Survived & $\begin{array}{l}\text { Non- } \\
\text { survived }\end{array}$ & Imported \\
\hline Wild & 2 & & 2 & & & & 2 & & \\
\hline Delta & 138 & & 50 & 72 & 15 & 1 & 130 & $\begin{array}{l}\text { 2 Malaysia, } \\
1 \text { Syri } \\
\text { lanka }\end{array}$ \\
\hline Omicron & 10 & 10 & & & & & 10 & & $\begin{array}{l}\text { 9 India, } \\
1 \text { Russia }\end{array}$ \\
\hline & 150 & & & & & & 142 & & \\
\hline
\end{tabular}

\section{2 | REFERENCES}

Brandal, L. T., MacDonald, E., Veneti, L., Ravlo, T., Lange, H., Naseer, U., Feruglio, S., Bragstad, K., Hungnes, O., Ødeskaug, L. E., Hagen, F., HanchHansen, K. E., Lind, A., Watle, S. V., Taxt, A. M., Johansen, M., Vold, L., Aavitsland, P., Nygård, K., \& Madslien, E. H. (2021). Outbreak caused by the SARS-CoV-2 Omicron variant in Norway, November to December 2021. In Eurosurveillance (Vol. 26, Issue 50, p. 2101147).

Hansen, C. H., Schelde, A. B., Moustsen-Helm, I. R., Emborg, H.-D., Krause, T. G., Mølbak, K., Valentiner-Branth, P., \& on behalf of the Infectious Disease Preparedness Group at Statens Serum Institut. (2021). Vaccine effectiveness against SARSCoV-2 infection with the Omicron or Delta variants following a two-dose or booster BNT162b2 or mRNA-1273 vaccination series: A Danish cohort study. MedRxiv, 2021.12.20.21267966. https://doi.o $\mathrm{rg} / 10.1101 / 2021.12 .20 .21267966$

Htun, Y. M., Win, T. T., Aung, A., Latt, T. Z., Phyo, Y. N., Tun, T. M., Htun, N. S., Tun, K. M., \& Htun, K. A. (2021). Initial presenting symptoms, comorbidities and severity of COVID-19 patients during the second wave of epidemic in Myanmar. Tropical Medicine and Health, 49(1), 62. https://doi .org/10.1186/s41182-021-00353-9

Lewnard, J. A., Hong, V. X., Patel, M. M., Kahn, R., Lipsitch, M., \& Tartof, S. Y. (2022). Clinical outcomes among patients infected with Omicron (B.1.1.529) SARS-CoV-2 variant in southern California. MedRxiv, 2022.01.11.22269045. https://doi. org/10.1101/2022.01.11.22269045

Public Health Ontario. (2021). COVID-19 Variant of Concern Omicron (B.1.1.529). https://www.publ ichealthontario.ca/-/media/documents/ncov/voc/202 2/01/covid-19-omicron-b11529-risk-assessment-jan -12.pdf?sc_lang $=$ en

Pyar, K. P., Hla, S., Min, A., Wunn, D., Aung, Z. N., Lin, M., Win, T., Aung, L., Kyaw, A., Ya,K., Tun, T., Kyaw, M., Oo, Z., Aung, Z., Lin, T., \& Htun, S. (2021). Breakthrough Infection among Fully Vaccinated Physicians Working 


\section{MEERP LTD}

in COVID- 19 Treatment Centers; Prevalence, Presenting Symp-toms, Co-Morbidities and Outcome in the Third Wave of Epidemics in Myanmar. Journal of Biomed-ical Research \& Environmental Sciences, 2, 721-730. https:// doi.org/10.37871/jbres1303

Sheikh, A., Kerr, S., Woolhouse, M., McMenamin, J., \& Robertson, C. (2021). Severity of Omicron variant of concern and vaccine effectiveness against symptomatic disease: National cohort with nested test negative design study in Scotland. University of Edinburgh Research Explorer. https://www.research .ed.ac.uk/en/publications/severity-of-omicron-varia nt-of-concern-and-vaccine-effectiveness-

UK Health Security Agency, (2021). SARS-CoV-2 variants of concern and variants under investigation in England. https://assets.publishing.service.gov.uk/ government/uploads/system/uploads/attachment_da ta/file/1045619/Technical-Briefing-31-Dec-2021-O micron_severity_update.pdf

Wang, L., Berger, N. A., Kaelber, D. C., Davis, P. B., Volkow, N. D., \& Xu, R. (2022). Comparison of outcomes from COVID infection in pediatric and adult patients before and after the emergence of Omicron. MedRxiv, 2021.12.30.21268495. https://d oi.org/10.1101/2021.12.30.21268495

Wei, C., Shan, K.-J., Wang, W., Zhang, S., Huan, Q., \& Qian, W. (2021). Evidence for a mouse ori- gin of the SARS-CoV-2 Omicron variant. BioRxiv, 2021.12.14.472632. https://doi.org/10.1101/2021.1 2.14.472632

World Health Organization, (2021). Update on Omicron. https://www.who.int/news/item/28-11-2021-u pdate-on-omicron

World Health Organization, (2022). COVID-19 vaccines. https://app.powerbi.com/view?r=eyJrIjoi MWNjNzZkNjctZTNiNy00YmMzLTkxZjQtNmJi ZDM2MTYxNzEwIiwidCI6ImY2MTBjMGI3LWJ kMjQtNGIzOS04MTBiLTNkYzI4MGFmYjU5MC IsImMiOjh9

Zoe Covid Study Group, (2022, January 18). What are the symptoms of Omicron? https://joinzoe.com/1 earn/omicron-symptoms

How to cite this article: K.P.P., K.K.S., K.W., Z.N.H.A., N.L.M., A.P.K., S.W.H., T.H.T., S.M.H., N.M.A., A.M.T., M.K.M., W.M., T.T.M., K.Z.Y., T.W., M.A.S., S.P.T., Y.L.A., Z.P.A., M.T.K., K.T.M., H.L.A. Initial presenting symptoms and severity of SARS-CoV-2 Wild type, the Delta variant and the Omicron variant infected cases in early fourth wave of epidemics in Myanmar. Journal of Medical Research and Health Sciences. 2022;1765-1769. https://doi.org /10.52845/JMRHS/2022-5-1-8 